INNOVACIÓN

\title{
Una experiencia para el desarrollo de actitudes del «trabajo en equipos de abogados» para la litigación oral y escrita a través del aprendizaje cooperativo en la asignatura de Derecho Internacional Privado
}

\author{
An experience for the development of attitudes of «work in teams of lawyers» for oral and \\ written litigation through cooperative learning in the subject of Private International Law
}

\author{
Odette Martínez PÉrez \\ Universidad de Oriente, Cuba
}

\section{Luis Alberto Alzate Peralta}

Instituto Superior Tecnológico Bolivariano de Tecnología, Ecuador

\begin{abstract}
RESUMEN El aprendizaje cooperativo es considerado por diversos autores como un método que permite desarrollar potencialidades para trabajar en equipo a través de una forma de organización social del grupo, la cual facilita que cada miembro del equipo despliegue habilidades, destrezas y valores basados en sus propias potencialidades, las mismas que han sido identificadas y maximizadas por el docente intencionadamente para garantizar una correcta cohesión de equipo. Se realizó una experiencia en la asignatura de Derecho Internacional Privado, en la cual se evidencia, según las valoraciones del equipo metodológico, que los estudiantes desarrollaron habilidades en el discurso oral y escrito, mejorando a su vez las relaciones de interdependencia con sus pares con habilidades desarrolladas. Ellos se mostraron más responsables por el proceso de la tarea, por lo que se formaron aspectos personológicos fundamentales en el ejercicio del derecho.
\end{abstract}

PALABRAS CLAVE Aprendizaje cooperativo, trabajo en equipos, Derecho Internacional Privado, litigación oral y escrita.

ABSTRACT Cooperative learning is considered by several authors as a method that helps to develop the potential to work as a team through a form of social organization of the group that allows each team member to develop capabilities, skills and values based on their own potentialities, the same which were identified and maximized by the teacher intentionally to ensure a correct team cohesion. An experience was realized 
in the subject of Private International Law, where there is evidenced, according to the assessments of the methodological team, that the students developed skills in oral and written speech, while improving the relationships of interdependence with their peers with developed skills. They were more responsible for the development of the task, so fundamental aspects were developed in the exercise of Law.

KEYWORDS Cooperative learning, teamwork, Private International Law, oral and written litigation.

\section{Introducción}

La educación superior en Cuba se transforma, desde 1990, al ritmo de las variables y novedosas demandas de la sociedad actual. En especial, para la Ciencia del Derecho, los cambios en el ejercicio y la enseñanza resultaron sustanciales, a la par de las transformaciones que en la economía y la política se sucedieron en la sociedad cubana tras la caída del campo socialista.

La visión que desde el derecho estatalista soviético tenían nuestras universidades había marcado la enseñanza con un carácter tradicionalista. La localización de caminos que permitieran la recuperación del país generaron nuevos derroteros en la formación de asesores y representantes de personas naturales y jurídicas. En una sociedad en la que se incrementó el ingreso de extranjeros a la nación, fundamentalmente en las categorías de visitantes y residentes temporales, comenzó a promoverse el desarrollo del turismo, la inversión extranjera, la colaboración económica y los planes de integración con América Latina y otras regiones, así como la reorientación de la política migratoria para los cubanos, con lo que se incrementaron las relaciones civiles, de propiedad, familia y comerciales con personas jurídicas y naturales extranjeras y cubanos residentes en el exterior y emigrados.

En este escenario, se produjo una transformación general de la Escuela de Derecho de la Universidad de Oriente de Santiago de Cuba y sus planes de estudio, potenciándose las raíces históricas y los estudios de Derecho Comparado, desde las experiencias de nuestros profesores en otras naciones. Ciertamente, el perfil de la carrera se amplió: hoy el nodo elemental de la formación son las habilidades para el ejercicio de la profesión en cualquier ley y nación, y el conocimiento de las diferentes instituciones de las ramas del Derecho, presentes o no en las normas jurídicas actuales, de modo que los futuros juristas puedan adaptarse a los constantes cambios legislativos y a ejercer en cualquier país del mundo a partir de la nueva política de cooperación en los servicios profesionales.

Por ello, es necesario que nuestros estudiantes se atemperen a las novedosas formas de organizar los recursos humanos en bufetes y consultorías en otros países, o sea, practicar la litigación oral y escrita en escenarios complejos y en equipos de abogados. 
El «trabajo en equipos de abogados» se entiende como la asociación de un grupo de profesionales del derecho para representar y asesorar a personas naturales y jurídicas en un proceso determinado, tomando diferentes roles para trabajar y llegar a una exitosa presentación en la corte.

Precisamente, el derecho internacional privado constituye una de las ramas de esta ciencia que mayor cambio experimentó y, contrario al resto de las disciplinas de estudios, hubo de incrementarse en su fondo de tiempo y objeto de enseñanza. No obstante, y a pesar de más de un siglo de estudio de la misma en Cuba, aún es insuficiente el aprovechamiento de sus potencialidades para el desarrollo de habilidades profesionales.

Por lo anterior, los profesores de Derecho Internacional Privado se insertan en diversos proyectos de investigación para solucionar estas insuficiencias y ofrecer una alternativa metodológica a la necesidad de formar la actitud para la «litigación oral y escrita en equipos de abogados». El presente artículo está dirigido a recrear la experiencia que a partir del aprendizaje cooperativo se desarrolla en la asignatura de Derecho Internacional Privado con alumnos de quinto año de la carrera en la Universidad de Oriente de Santiago de Cuba para este último fin.

\section{El aprendizaje cooperativo como método para trabajar actitudes del «trabajo en equipos de abogados»}

Entendemos por «métodos docentes» un conjunto de decisiones sobre los procedimientos a emprender y sobre los recursos a utilizar en las diferentes fases de un plan de acción que, organizados y secuenciados coherentemente con los objetivos pretendidos en cada uno de los momentos del proceso, nos permiten dar una respuesta a la finalidad última de la tarea educativa, desde el propio proceso de enseñanza aprendizaje, teniendo en cuenta la ley de la unidad de la instrucción y la educación (De Miguel Díaz, 2005: 83).

Los métodos dentro del proceso de enseñanza aprendizaje se desarrollan en dos áreas: las actividades presenciales, que reclaman una intervención directa de profesores y alumnos; y las modalidades no presenciales, en las que se engloban las actividades que los alumnos puedan realizar libremente, ya sea en forma individual o por trabajo en grupo (Pinto, 2014). Existe una gran variedad, aunque entre los principales podemos mencionar los siguientes:

- Método expositivo o lección magistral, cuya finalidad es transmitir conocimientos y activar procesos cognitivos en el estudiante.

- Estudio de casos, cuyo objeto es la adquisición de aprendizajes mediante el análisis de casos reales o simulados.

- Resolución de ejercicios y problemas, con el que se pretende ejercitar, ensayar 
y poner en práctica los conocimientos previos.

- Aprendizaje basado en problemas (ABP), que persigue desarrollar aprendizajes activos a través de la resolución de problemas (Clough y Shorter, 2015; Orji, 2015).

- Aprendizaje orientado a proyectos, que tiene por finalidad la realización de un proyecto para la resolución de un problema aplicando habilidades y conocimientos adquiridos.

- Aprendizaje cooperativo, cuya finalidad es desarrollar aprendizajes activos y significativos de forma cooperativa (Duch, 2006: 56-61; Hernández Trasobares y Lacuesta Gilaberte, 2007: 32).

Los estudios sobre aprendizaje cooperativo datan de la década de 1970 y son múltiples los autores que han abordado las experiencias del aprendizaje fomentando las relaciones de cooperación que se dan en el aula. En el mundo anglosajón es investigado el aprendizaje cooperativo, donde se lo considera como un enfoque de investigación grupal (Hernández Trasobares y Lacuesta Gilaberte, 2007: 32) cuyas bases teóricas están en las concepciones de Dewey y Lewin, en la psicología constructivista de la cognición y en la teoría de la motivación intrínseca para aprender.

Los psicólogos sociales norteamericanos David y Roger Johnson (Johnson y Johnson, 1990), al explicar qué es el aprendizaje cooperativo, plantean que es trabajar juntos para cumplir con determinadas metas compartidas, con el uso instructivo de pequeños grupos, para que los estudiantes trabajen unidos, lo que maximiza la posibilidad de aprendizaje de cada uno. Los alumnos tienen dos responsabilidades: aprender el material asignado y asegurarse de que todos los demás miembros del grupo lo hagan. Estos autores señalan que los elementos básicos del aprendizaje cooperativo son:

- Interdependencia positiva: Los estudiantes tienen que concientizar que están vinculados unos a otros de una forma tal que no pueden lograr el éxito individual si los demás no lo logran, lo que supone compartir recursos, objetivos comunes y roles específicos para cada uno.

- Favorecimiento de la interacción cara a cara: Cuando los estudiantes explican oralmente uno al otro cómo resolvieron los problemas, discuten con los demás la naturaleza de los conceptos y las estrategias aprendidas, trasmiten conocimientos a sus compañeros y explican los vínculos entre lo ya aprendido con lo nuevo.

- Responsabilidad individual: Cada miembro del grupo debe responsabilizarse personalmente de su propio trabajo, de los resultados a los que llegue y, en consecuencia, de sus aportaciones al grupo. 
- Habilidades sociales de intercambio interpersonal en pequeños grupos: Los grupos no funcionan si no poseen o utilizan el liderazgo, la toma de decisiones, la comunicación, las habilidades para manejar conflictos y la creación de confianza. Son habilidades académicas de acuerdo con las características del aprendizaje cooperativo.

- Conciencia del propio funcionamiento como grupo: En lo que se ha realizado, lo que falta por hacer, lo que salió como esperaba, en cómo relacionarse, etcétera.

Los presupuestos del aprendizaje cooperativo dan la posibilidad de que el estudiante pueda intercambiar reflexivamente con sus compañeros, colaborar en el trabajo, compartir ideas con los más experimentados y atreverse a plantear nuevas ideas, lo que resulta esencial para el trabajo con supuestos de derecho, que esencialmente suponen la discusión o análisis de un caso o situación jurídica, entendida la misma como un hecho de la vida que tiene un impacto para el surgimiento o cese de derechos, deberes, facultades y responsabilidades (Iborra Cuéllar e Izquierdo Alonso, 2010; Fernández March, 2005; Palazón y otros, 2011; García-Merino, Urionabarrenetxea y Bañales-Mallo, 2016).

La cooperación permite la integración de conocimientos, el enriquecimiento o simplificación de acciones y orientaciones valorativas más humanas como fuente de interacción, la consideración del criterio del otro, la autorregulación y la toma de conciencia en cuanto a cómo ampliar su sistema referencial.

Lo anterior llama a la necesidad de reconsiderar el proceso formativo del jurista a través del aprendizaje cooperativo situando el proceso de enseñanza aprendizaje como el principal objeto de cambio, y a reconceptualizar el aprendizaje en el contexto de las Ciencias Jurídicas, para recrear las más modernas formas de organización laboral de los recursos humanos en el mundo del derecho (Orji, 2015), a través de la organización de los equipos para el desarrollo de las tareas en el aula, lo cual propicia el crecimiento de las cualidades profesionales necesarias para su futuro desempeño profesional.

El aprendizaje cooperativo tiene un amplio desarrollo teórico y los resultados de su implementación práctica dan cuenta de una forma de organización social que fomenta niveles superiores de desarrollo socioeducativo. Esta premisa se inserta en el proceso de enseñar y aprender, que el docente debe tener en cuenta para contribuir al desarrollo del estudiante y su preparación para enfrentar la profesión.

Añádase a lo anterior que la solución de la tarea grupal es favorecida por la creación, en la dinámica de trabajo de la asignatura, de constantes situaciones de cooperación y la posibilidad de que los estudiantes aprendan a cooperar entre sí y que vayan desarrollando las habilidades necesarias que les permitirá resolver la tarea, recreándose en el transcurso de la asignatura y, según lo descrito en la literatura cientí- 
fica (Pedraz, 2004: 11; Roa Banquez y Romero Gómez, 2009: 12; Loyens y otros, 2015; Hmelo-Silve, 2004), las condiciones que permitan trabajar en un ambiente cooperado.

No obstante, para el desarrollo de la cooperación (Johnson y Johnson, 1990: 6880), los estudiosos han expuestos factores claves o necesarios que el profesor debe implementar, como son: la distribución y asignación de roles a los alumnos, la estructura interna de la tarea propuesta y la delimitación de las formas de apoyo que ofrece el profesor a lo largo de la actividad.

Sobre la distribución y asignación de roles, es vital que el docente no deje a los grupos crearse de manera espontánea, sino que se realice su planificación equilibrando sus componentes, integrando estudiantes con diferentes posibilidades de asimilación del contenido y aquéllos más dispuestos a cooperar con los que no lo están, previa valoración del nivel de desarrollo ontogenético de los estudiantes, siempre potenciando la necesidad de que aprendan a trabajar en grupo, sobre la base de tareas típicamente grupales y que no puedan llegar a ser resueltas individualmente (Andreu-Andrés, 2016).

Asimismo, debe apercibirse a los estudiantes que para el desarrollo de la cooperación en el seno del grupo son necesarios los siguientes presupuestos (Pedraz, 2004: 15; Roa Banquez y Romero Gómez, 2009: 18): ponerse de acuerdo sobre lo que hay que realizar, decidir cómo se hace y qué va a hacer cada cual, realizar los correspondientes trabajos o pruebas individuales, considerar cómo se complementa el trabajo, ejecutar individualmente cada una de las partes a medida que se van analizando las diversas realizaciones en función de criterios preestablecidos por el profesor o por el propio grupo y, finalmente, valorar en el grupo los resultados en función de los criterios asumidos o explicitados con anterioridad.

Sobre la estructura interna de la tarea propuesta (De Miguel Díaz, 2005: 92), deben tratar de conjugar diferentes tipos de información. En su diseño deben demandar la coordinación, comparación y análisis de la información, estableciéndose relaciones y llegando a la síntesis de la misma; y deben promover resolver problemas, confrontar puntos de vista y valores, desarrollar o demostrar modelos conceptuales, de procedimientos y de procesos, organizar y elaborar esquemas de actuación, explicaciones y principios.

El docente debe prestar especial atención a estos detalles durante la clase cooperativa en el funcionamiento del grupo, resumiendo lo anterior desde la perspectiva de valoración del objetivo principal de la actividad de grupo (interacción social o aprendizajes formales) y de la pertinencia de la situación grupal y la supervisión del proceso de interacción (Pedraz, 2004: 34).

Desde el punto de vista didáctico se recomiendan las siguientes actividades para estructurar una clase cooperativa (Johnson, Johnson y Holubec, 1999):

- Especificar claramente los objetivos de la clase, no sólo de tipo instructivos, sino también las habilidades de colaboración necesarias de desarrollar por los alumnos. 
- Tomar una serie de decisiones acerca del lugar de los estudiantes en el aprendizaje grupal antes de comenzar la clase; el tamaño del grupo deberá ser de dos a seis integrantes.

- Asignación de los estudiantes por grupos. Estos serán homogéneos cuando se trabaje con una habilidad específica, un procedimiento o un grupo de hechos, pero cuando se trabaje en la resolución de problemas o sobre conceptos básicos de aprendizaje los grupos deberán ser heterogéneos.

- Planificar qué tiempo deben trabajar unidos, lo que deberá no ser menos de tres semanas, aunque puede variar por diversos criterios.

- Preparar el aula para que los estudiantes puedan sentarse lo suficientemente cerca para compartir los materiales docentes, hablar tranquilamente y mantener el contacto visual con los demás, el círculo es lo mejor y el profesor debe acceder a cada grupo con facilidad.

- Planificación de los materiales de estudio, que deben ser distribuidos entre sus miembros de forma tal que cada uno tenga una parte para poder completar las tareas entre todos.

- Asignación de roles. La interdependencia cooperativa puede llevarse a cabo a través de la asignación de roles complementarios e interrelacionados a los miembros del grupo.

Tales roles pueden ser: resumir la mejor conclusión o la mejor respuesta, chequear que todos los miembros puedan explicar cómo se llegó a una respuesta o a una conclusión, precisar cualquier error en la explicación o el resumen y pedirle a los demás que relacionen los nuevos conceptos y estrategias con el material estudiado previamente.

Existen distintas formas de organizar el trabajo cooperativo, pudiendo hablar de grupos informales, grupos formales y grupos base: los dos primeros pueden darse en una asignatura sin problemas, mientras que el grupo base sólo acaecerá cuando se organice institucionalmente la enseñanza en aprendizaje cooperativo. Por ello no se particularizará sobre el mismo en este artículo. Solamente nos limitamos a exponer el que se ha utilizado en nuestra experiencia, el aprendizaje cooperativo informal, como aquél que se organiza de forma temporal, no tiene solución de continuidad y se forma habitualmente para llevar a cabo una determinada tarea académica en el transcurso de una clase, con pequeños grupos de aprendizaje de cuatro a seis alumnos de carácter heterogéneo, con la firme idea de que los estudiantes aprenden mejor cuando están trabajando juntos que cuando están atentos a lo que dice y hace un profesor. 


\section{El Derecho Internacional Privado como escenario para el desarrollo del método del aprendizaje cooperativo}

La asignatura de Derecho Internacional Privado se encuentra inserta en la disciplina de derecho internacional, y es una de las materias que distingue la impartición del derecho en nuestro país desde hace más de un siglo, llegando a reconocerse a la Escuela de La Habana como una de las de mayor renombre a partir de la obra del jurista Antonio Sánchez de Bustamante y Sirvén.

En la Universidad de Oriente se imparte desde la fundación de la carrera. De ese modo, la incidencia de esta materia en la formación del profesional del derecho es un imperativo que se funda en la clara tendencia a la internacionalización de las relaciones sociales en todos los órdenes.

Es una asignatura en la que se estudian las relaciones privadas de carácter civil, propiedad, familia y comercial, donde intervienen personas naturales o jurídicas extranjeras y se realizan fuera del país o por residentes en el exterior. Pretende satisfacer la necesidad de formar a los juristas en los principios, categorías y sistema de relaciones sobre los cuales se erige la legislación que regula las relaciones anteriores y las cuestiones de migración y extranjería, lo que le posibilita interpretar y aplicar esa legislación para la preservación de los derechos adquiridos por las personas naturales y jurídicas implicadas en éstas, en consonancia con los intereses económicos, políticos y sociales de los Estados y los principios de la comunidad jurídica internacional.

Con el estudio del derecho internacional privado, el alumno se enfrenta por primera vez a los problemas típicos que engendran las relaciones jurídicas privadas cuando alguno de sus elementos sea extranjero, lo que implica un contacto con sistemas jurídicos diferentes y, por ende, la posible intervención de foros extranjeros y la aplicación de leyes de distintos países a aquél en el cual se realiza o se conoce dicha relación.

La asignatura, con un enfoque teórico-práctico, se compone de siete temas divididos en parte general y parte especial, agrupados por los elementos comunes que realmente registran, y distribuyéndose su fondo horario en conferencias, seminarios, talleres y clases prácticas. Los seminarios están repartidos en casi todos los temas, y se dedican aspectos temáticos de importancia teórica y práctica. Las clases prácticas se emplean en la parte general, pero cobran exclusivo énfasis en la parte especial, a fin de que el alumno, al concluir el estudio de la asignatura, haya desarrollado las habilidades necesarias para dar solución a casos de derecho internacional privado, con independencia de la naturaleza jurídica de la ley que le ha dado origen a los mismos.

Su fin u objetivo formativo dentro del plan de estudio es solucionar de forma individual y en equipo, con un nivel creativo, casos prácticos vinculados a las relaciones privadas de carácter civil, propiedad, familia y comercial, en los que intervienen personas naturales o jurídicas extranjeras, se realizan fuera del país o por residentes 
en el exterior, para lograr un jurista competente, solidario y justo en el campo de las relaciones internacionales.

Como rama integradora, se consolidan habilidades profesionales como:

- Representar a personas naturales y jurídicas en actos fundacionales, conciliaciones, arbitrajes, convenciones, negociaciones y actos jurídicos en general precisando, en consonancia con sus respectivos momentos de desarrollo, sus exigencias de forma, tiempo, lugar y autoridad o funcionario correspondiente, así como los fundamentos jurídico-materiales de su actuación y con dominio del vocabulario técnico pertinente.

- Decidir conflictos jurídicos partiendo de los fundamentos técnicos y éticos de la asesoría y la representación legal, pero conscientes del alto sentido de responsabilidad que entrañan estas funciones, en los que la necesidad de lograr una adecuada armonía entre el respeto a los derechos y garantías de los ciudadanos y la protección de los intereses sociales exigen una mayor proyección humanista, ética, social y política.

- Elaborar documentos legales con los requisitos para surtir efectos extraterritoriales, así como valorar los documentos legales extranjeros y dictaminar sobre su posible aceptación y aplicación en nuestro país.

Además, la asignatura constituye una materia en la que el estudiante deberá desarrollar consolidar valores sobre la base de tres dimensiones: intelectual, estética y ética. La primera dimensión se vincula con la formación del valor saber, que enmarca a su vez tres elementos: rigor científico, responsabilidad y honestidad. Por su parte, la dimensión estética formará los valores de creatividad, dignidad profesional y sensibilidad, calidad del discurso jurídico, dignidad en su porte y aspecto y sobriedad en el vestir. Por último, la dimensión ética formará los valores de la dignidad, discreción, el humanismo, la honestidad, la probidad, cortesía y respeto en sus relaciones, independencia, solidaridad, sentido de la justicia y la equidad. En esta dimensión se trabaja el cooperativismo como valor que sustenta el trabajo en equipos de abogados.

\section{La implementación del aprendizaje cooperativo en la asignatura de Derecho Internacional Privado para trabajar actitudes de «trabajo en equipos de abogados"}

Con la introducción del plan de estudio $\mathrm{D}$, su perfeccionamiento y la reciente proyección del plan E, se plantea la posibilidad de adecuar en el mismo la docencia de las clases prácticas y talleres de la asignatura, que consistían en la discusión y resolución de casos, aunque también tenían lugar en ellas el estudio de las fuentes clásicas y la estructura de las fórmulas procesales, en las que primaba la tendencia individualista 
en el aprendizaje y los alumnos, si bien trabajaban en equipo, se organizaban fundamentalmente para la investigación y la solución de casos, desde una concepción del aprendizaje colaborativo, no cooperativamente, que es el que desarrolla la nueva tendencia de trabajo en equipo de abogados para la solución de un caso, porque no se trata de ayudar al otro, sino que consiste en que cada quien posea una tarea que tribute a la solución colectiva del problema, incluso en un mismo modo de actuación profesional.

En este contexto, y dado que de alguna manera ya habíamos venido utilizando una metodología similar al aprendizaje por problemas, nos decidimos por ensayar el método cooperativo para el estudio de casos. Para ello, se decidió que la materia más adecuada para que los alumnos pudieran enfrentarse a este nuevo método era la solución de casos prácticos integradores de civil, mercantil y familia internacional en las formas organizativas docentes de talleres y clases prácticas, en los modos de actuación profesional de representación o abogacía, administración de justicia o jueces y control de la legalidad o fiscalía, desarrollando las habilidades ya comentadas.

Asimismo, se prepararon las condiciones para la implementación del mismo con determinadas acciones en todas las clases, pero preferentemente en algunos espacios, como la conferencia introductoria de la asignatura. En el primer tema, se le explica a los estudiantes los diferentes modos de actuación y las peculiaridades de su ejercicio laboral individual y en equipos en el campo de derecho internacional privado; se prosigue el debate en el seminario primero, con el fin de que valoren la importancia de litigar en equipos, y se explica la metodología utilizada para ello.

Por su parte, en el tercer tema de competencia judicial internacional se trabaja la habilidad de redacción de actos procesales (son los actos orales y escritos que conforman el proceso, como por ejemplo la demanda, la contestación, la vista oral, la prueba de testigo) en materia de derecho internacional privado y se les enseña las peculiaridades de los escritos de alegaciones (demanda y contestación, donde cada parte alega los hechos de los que va a defenderse, alegar y las normas que va a utilizar), proposición de pruebas (escrito en que se proponen las pruebas de que cada parte intenta valerse) y las sentencias con el fallo de los jueces. Se realiza esta habilidad en clases prácticas y talleres, en los que se ejercitan el discurso jurídico en los actos procesales orales (vistas, donde las partes ilustran al tribunal de sus alegaciones de hecho; derecho sobre la cuestión general del proceso, valorándolas a la luz de las pruebas, y comparecencias, donde se ventila una cuestión particular dentro de la general del proceso que índice directamente en este, valorándolas a la luz de los hechos, los fundamentos de derechos y las pruebas).

El paso siguiente fue organizar los grupos dentro de la clase. Creamos grupos de nueve a doce estudiantes: seis que trabajaron la abogacía, constituyéndose en dúos de contraparte, tres que se establecieron como jueces y tres en el rol de fiscales civiles. Los grupos se conformaron atendiendo al diagnóstico inicial del profesor principal de año 
y teniendo en cuenta sus niveles de aprovechamiento académico precedente, socialización y hasta ese momento el logro en su formación de los valores que exige el modelo del profesional, para insistir en el trabajo con ellos según este diagnóstico inicial.

Organizados los grupos, se procede a la asignación de los roles a cada uno de los integrantes del mismo en el caso y a la distribución de la tarea según la metodología para el «trabajo en equipos de abogados», que consiste en la presentación del caso por el responsable del bufete, discusión, análisis del caso, búsqueda de pruebas, discusión final, redacción de los escritos y presentación a la corte, defensa oral de las mismas ante la corte y fallo del juez escrito y oral.

La primera tarea que se asigna a cada uno de los estudiantes es lo que llamamos el trabajo individual, que consiste en el estudio y análisis del material asignado, búsqueda de la legislación y posibles recursos de prueba. Una vez finalizada esta tarea, se constituye nuevamente los subgrupos de tres para que, desde su modo de actuación profesional, preparen su rol, o sea, el equipo de abogados que demanda prepara sus escritos y alegaciones y lo trasladan al equipo de la contraparte; éstos realizan lo mismo y se da entre ellos el intercambio, como si estuvieran en un debate procesal real. Terminada la fase de alegaciones se trasladan, finalmente, al equipo que juega el rol de justicia para dictar el fallo.

Como las fases de la tarea se corresponden con los actos procesales de los diferentes procesos, cada vez que se termina uno de ellos, el profesor debate sobre el contenido final y cómo se desarrolló el trabajo de equipo. Se presenta, finalmente, una simulación de vista pública o comparecencia según el proceso que sea, que ilustra al resto del aula de clases del caso cómo ha sido trabajado en su desarrollo y su solución, así como la manera en que se ha trabajado en el equipo, como acto oral resumen.

La forma de evaluar el rendimiento de los alumnos en el taller final, que antes era la de someterlos a la realización de una prueba escrita, se cambió por un taller en el que se realizaba el mismo ejercicio entre los equipos, pero sin la ayuda del docente.

Este sistema tiene beneficios, como que los estudiantes aprenden a enfrentarse al estudio de una materia no sólo para su comprensión a nivel individual, sino para hacerla comprensible a los demás compañeros; por otra parte, les sirve para adquirir destrezas en la expresión oral y escrita. Además, los alumnos aprenden a trabajar en grupo, con lo que ello supone aprender a escuchar al otro, rebatir argumentos y asumir sus responsabilidades frente a los demás.

Lo anterior fundamenta la utilización del aprendizaje cooperativo como método en la enseñanza del derecho internacional privado y del derecho en universidades foráneas. Se ha utilizado el mismo método en el entrenamiento de las habilidades prácticas; sin embargo, en Cuba no ha sido difundido su empleo y otros elementos para transformar la enseñanza de las ciencias jurídicas y avanzar hacia donde lo exigen las demandas sociales y las más modernas tendencias para el trabajo de casos en el derecho. 


\section{Conclusiones}

Esta experiencia piloto ha sido desarrollada en el seno del aula, concretamente en la asignatura de Derecho Internacional Privado, en su carácter de asignatura integradora de la carrera, a partir del «aprendizaje cooperativo informal», lo cual viene a referirse al hecho consistente en organizar, de forma temporal, el aprendizaje cooperativo con la finalidad de llevar a cabo una determinada tarea en el transcurso de varias clases.

Como resultados de la experiencia, corroborados por la entrevista semiestructurada a estudiantes y a tres controladores que realizaron inspecciones docentes a la profesora, pueden sistematizarse las siguientes:

- Desde la perspectiva del docente, la enseñanza permite un aprendizaje con mayor profundidad y concreción que en el supuesto del empleo de otras metodologías docentes.

- El alumno adquiere mayor responsabilidad individual en su proceso de aprendizaje, tanto en el estudio del propio material y el propuesto por el docente en cada caso; la materia se aprehende por el alumno en mejor medida y se trabajan por cada una de las habilidades profesionales.

- Con este sistema de aprendizaje se pretende la consecución de diferentes valores, como responsabilidad, honestidad, creatividad, dignidad profesional y sensibilidad; calidad del discurso jurídico, dignidad en su porte y aspecto, sobriedad en el vestir, dignidad, discreción, humanismo, probidad, cortesía y respeto en sus relaciones, independencia, solidaridad, sentido de la justicia y la equidad.

- Se potencia competencias transversales, como pueden ser la obtención de habilidades en las relaciones interpersonales, la capacidad de organización y planificación y la capacidad de la gestión de la información; relaciones interpersonales por lo que a la pertenencia a un grupo se refiere, así como la toma de conciencia de que existe un grupo al que pertenece uno de esos miembros; capacidad de organización y planificación, en la medida en que los miembros de cada uno de los subgrupos configurados en el seno del grupo original deben distribuirse el material aportado por el docente y aprender a planificar cada una de las actividades que el grupo va a desarrollar.

- Por último, por lo que se refiere a la gestión de la información, son los estudiantes quienes reciben la información por parte del docente, amplían los conocimientos y presentan un esquema común a todos ellos, esquema consensuado al resto del grupo originario. 


\section{Referencias}

ANDReu-Andrés, María Ángeles (2016). «Aprendizaje cooperativo o colaborativo: ¿Hay alguna diferencia en la percepción de los estudiantes universitarios?». Revista Complutense de Educación, 27 (3): 1.041-1.06o. DOI: 10.5209/rev_RCED.2016. v27.n3.47398.

Clough, Joanne y Gillian Shorter (2015). «Evaluating the effectiveness of problembased learning as a method of engaging year one Law students». Law Teacher, 49 (3): 277-302. DOI: 10.1080/03069400.2015.1011926.

Duch, Bárbara (2006). «Modelos para la instrucción basada en problemas en cursos de pregrado». En Bárbara Duch, Susan Groh y Deborah Allen (editoras), El poder del aprendizaje basado en problemas. Una guía práctica para la enseñanza universitaria. Trad. de César Mendoza Loyola. Lima: Ediciones Pontificia Universidad Católica del Perú.

Fernández March, Amparo (2005). Nuevas metodologías docentes. Valencia: ICE, Universidad Politécnica de Valencia.

García-Merino, José Domingo, Sara Urionabarrenetxea y Amaia BañalesMallo (2016). «Cambios en metodologías docentes y de evaluación: ¿Mejoran el rendimiento del alumnado universitario?». Revista Electrónica de Investigación Educativa, 18 (3): 1-18. Disponible en http://bit.ly/2zVwBoz.

Hernández Trasobares, Alejandro y Raquel Lacuesta Gilaberte (2007). «Aplicación del aprendizaje basado en problemas (PBL) bajo un enfoque multidisciplinar: Una experiencia práctica». En Juan Carlos Ayala Calvo (coordinador), Conocimiento, innovación y emprendedores: Camino al futuro (pp. 30-43). La Rioja: Ediciones Universidad de La Rioja.

Hmelo-Silve, Cindy (2004). «Problem-based learning: What and how do students learn?». Educational Psychology Review, 16 (3): 235-266. DOI: 10.1023/B:EDPR.0oooo34022.16470.f3.

Iborra Cuéllar, Alejandro y Mónica IzQuierdo Alonso (2010). «¿Cómo afrontar la evaluación del aprendizaje colaborativo? Una propuesta valorando el proceso, el contenido y el producto de la actividad grupal». Revista General de Información y Documentación, 20: 221-241. Disponible en http://bit.ly/2zT306s.

Johnson, David y Roger Johnson (1990). What is cooperative learning? Perspectives on small group learning. Ontario: Rubicon Publishing.

Johnson, David, Roger Johnson y Edythe Holubec (1999). El aprendizaje cooperativo en el aula. Buenos Aires: Paidós.

Loyens, Sofie, Suzanne Jones, Jeroen Mikkers y Tamara van Gog (2015). «Problembased learning as a facilitator of conceptual change». Learning and Instruction, 38 : 34-42. DOI: 10.1016/j.learninstruc.2015.03.002. 
De Miguel DíAz, Mario (director) (2005). Modalidades de enseñanza centradas en el desarrollo de competencias. Oviedo: Ministerio de Educación y Ciencia, Universidad de Oviedo.

Palazón, Alfonso, María Gómez, Juan Cándido Gómez, María Concepción Pérez y Juan GómEZ (2011). «Relación entre la aplicación de metodologías docentes activas y el aprendizaje del estudiante universitario». Bordón, 63 (2): 27-40. Disponible en http://bit.ly/2zTxEyl.

OrJI, Peter (2015). «Problem-based approach in property law: A university's strategy in focus». Law Teacher, 49 (3): 372-387. DOI: 10.1080/03069400.2015.1040295.

Pedraz, Azucena (coordinadora) (2004). «Un método de aprendizaje para toda la vida: $\mathrm{El} \mathrm{ABP}$ en el espacio europeo de la educación superior». En El aprendizaje basado en problemas: Una herramienta para toda la vida. Madrid: Agencia Laín Entralgo, 2004.

PinTo, Yenny (2014). «El aprendizaje cooperativo como metodología para la formación por competencias en la carrera de Derecho». Revista Pedagogía Universitaria $y$ Didáctica del Derecho, 1 (2): 69-79. Disponible en http://bit.ly/2zTzX4t.

RoA BANquez, Katherine y Alexander Romero Gómez (2009). «Construcción de conocimiento con metodología de aprendizaje basado en problemas frente al modelo tradicional, en ambiente virtual». Teoría y Praxis Investigativa, 4 (1): 11-23. Disponible en http://bit.ly/2zTtF 4 K.

\section{Sobre los autores}

Odette Martínez Pérez es doctoranda en Ciencias Jurídicas, máster en Derecho Constitucional y Administrativo y profesora auxiliar de la Universidad de Oriente, Cuba.Su correo electrónico es odettem@uo.edu.cu.

Luis Alberto Alzate Peralta es doctorando en Ciencias Pedagógicas, máster en Educación Médica y profesor titular del Instituto Superior Tecnológico Bolivariano de Tecnología, Ecuador. Su correo electrónico es lalzate@bolivariano.edu.ec. 


\title{
REVISTA PEDAGOGÍA UNIVERSITARIA Y DIDÁCTICA DEL DERECHO
}

La Revista Pedagogía Universitaria y Didáctica del Derecho (RPUDD) es una publicación científica semestral que contribuye tanto a la reflexión multidisciplinaria sobre Pedagogía Universitaria y Didáctica del Derecho, para la formación y consolidación de esta área de investigación, como a la difusión de prácticas innovadoras en la enseñanza-aprendizaje del Derecho, considerando el contexto nacional e internacional..

\author{
DIRECTORA \\ María Francisca Elgueta Rosas \\ ASISTENTE EDITORIAL \\ Priscilla Saavedra Medina \\ SITIO WEB \\ pedagogiaderecho.uchile.cl \\ E-MAIL \\ rpedagogia@derecho.uchile.cl \\ LICENCIA DE ESTE ARTÍ́CULO
}

Creative Commons Atribución Compartir Igual 4.0 Internacional

\author{
9 \\ La edición de textos, el diseño editorial \\ y la conversión a formatos electrónicos de este artículo \\ han estado a cargo de Tipográfica \\ (www.tipografica.cl).
}

